www.nature.com/ejhg

\title{
Marfan syndrome in the third Millennium
}

\author{
Gwenaëlle Collod-Béroud $^{1}$ and Catherine Boileau ${ }^{*, 1,2}$
}

${ }^{1}$ INSERM U383, Université Paris V, Hôpital Necker-Enfants Malades, 149-161 rue de sèvres, 75743 Paris Cedex 15, France; ${ }^{2}$ Laboratoire de Biochimie, d'Hormonologie et de Génétique Moléculaire, Hôpital Ambroise Paré AP-HP, 9 av Charles de Gaulle, 92104 Boulogne Cedex, France

The Marfan syndrome (MFS) is a prominent member of heritable disorders of connective tissue with manifestations involving primarily the skeletal, ocular and cardiovascular systems but also and less systematically investigated the lung, skin and integument, and dura. Over the last two decades, a considerable amount of clinical, molecular and protein data had accumulated. In combination with the study of natural and transgenic animal models, this new information provides greater insight into the pathogenic mechanisms underlying not only the pleiotropic manifestations of MFS but also the important degree of clinical variability (age of onset and severity) observed between patients. The following aspects will be described in this review: the structure and function of fibrillin-1; the fibrillin proteins; mutations in the FBN1 gene and pathogenic mechanisms; animal models. Finally, the currently available laboratory diagnostic tests and their limits will be discussed.

European Journal of Human Genetics (2002) 10, 673-681. doi:10.1038/sj.ejhg.5200876

Keywords: Marfan syndrome; MFS; FBN1; fibrillin-1; review; structure

\section{Introduction}

A hundred years have now elapsed since Dr Antonin Marfan $^{1}$ reported on the case of Gabrielle P thus describing some of the skeletal features that today define the syndrome that carries his name. Since then, substantial progress has been made with respect to the description of the pleiotropic manifestations of this disease, the understanding of underlying pathophysiological mechanisms and the availability of prevention and treatment of major complications.

\section{Nosology: What is Marfan syndrome today?}

Marfan syndrome (MFS, OMIM 154700) is an autosomal dominant connective tissue disorder that has an estimated incidence of $1 / 5000$ with probably over $25 \%$ of sporadic cases. The syndrome involves many systems (skeletal, ocular, cardiovascular, pulmonary, skin and integument, and dura) but its more prominent manifestations are skeletal, ocular and cardiovascular. In 1986, an international group of experts agreed upon diagnostic criteria to distin-

*Correspondence: C Boileau; INSERM U383, CHU Necker-Enfants Malades, Clinique Maurice LAMY, 149-161, rue de sèvres, 75743 Paris Cedex 15, France. Tel: 3314449 4485; Fax: 3314783 3206;

E-mail: boileau@necker.fr

Received 30 January 2002; revised 16 July 2002; accepted 18 July 2002 guish classic Marfan syndrome from many related disorders. These criteria constitute what is currently referred to as the 'Berlin nosology'. ${ }^{2}$ Patients are diagnosed based on involvement of the skeletal system and two other systems with at least one major manifestation (ectopia lentis, aortic dilation/dissection, or dural ectasia). Patients with an affected first degree relative are required to have involvement of at least two other systems with one major manifestation preferred but not required.

This nosology has been found wanting in many individual cases, and revised criteria were subsequently proposed that constitute the 'Ghent nosology'. ${ }^{3}$ This new formulation requires involvement of three systems with two major diagnostic manifestations. It provides for major skeletal manifestations and considers affected first-degree relatives or molecular data as major diagnostic criteria.

Finally, development of preventive measures and surgery for aortic aneurysms and dissection have lead to treatment of life-threatening cardiovascular complications associated with the Marfan syndrome and have considerably altered life expectancy for patients. Interestingly, the review of the medical problems of surviving patients has revealed possible unidentified pleiotropic manifestations of the Marfan syndrome or manifestations that could be related to aging of this population. These medical problems 
include the onset of arthritis at an early age, varicose veins, ruptured or herniated discs, and prolapse of the uterus or bladder in women. These medical problems now need to be properly investigated and monitored.

The continued efforts to redefine diagnostic criteria emphasize persistent shortcomings. The phenotype of the Marfan syndrome remains incompletely defined. Most manifestations are age-dependent and are difficult to quantify. The Ghent nosology has been field-tested in The National Institutes of Health. ${ }^{4}$ Their study shows that $19 \%$ of patients diagnosed under the Berlin criteria failed to meet the Ghent standard. Molecular data are important to better characterise this subset and to study its natural history.

\section{The Marfan syndrome and FBN1}

Scientists, as early as 1931, suggested that the basic defect in Marfan syndrome lay in a defect in the mesoderm. ${ }^{5}$ In 1955, Victor McKusick considered the syndrome as a prominent member of the new nosologic group he named 'the heritable disorders of connective tissue'. ${ }^{6}$ The Marfan syndrome was long considered to be due to a defect either in one of the collagens or elastin since abnormalities in their fibres were reported in affected subjects. However, protein and gene studies conclusively demonstrated that neither was involved. In 1986, Sakai and co-workers identified a new extracellular matrix protein that they named 'fibrillin'7 (OMIM 134797). This protein is the major component of microfibrils, structures found in the extracellular matrix either as isolated aggregates or closely associated with elastin fibres. Ultrastructurally, microfibrils display a typical 'beads-on-a-string' appearance consisting of a long series of globules connected by multiple filaments. In 1990, Hollister et al., using a monoclonal antibody against fibrillin, reported abnormalities of the microfibrillar system in the Marfan syndrome. ${ }^{8}$ The following year, the gene encoding fibrillin-1 (FBN1) was cloned and the first mutations in the gene were identified in Marfan syndrome patients. ${ }^{9-11}$ Interestingly, the year before the FBN1 gene was cloned, Kainulainen et al., ${ }^{12}$ demonstrated through linkage analysis that the gene involved in classic complete forms of the Marfan syndrome was located on human chromosome 15 precisely where the FBN1 gene was later located. Therefore the identification of the gene defect in Marfan syndrome is a rare example in which both positional and functional cloning strategies converged rapidly to identify a disease gene.

\section{The FBN1 gene and other members of the fibrillin family}

The gene encoding type 1 fibrillin ( $F B N 1$ ) lies on the long arm of chromosome 15 at $15 q 15-q 21.1$. This very large gene (first estimated at $110 \mathrm{~kb}$, now at over $230 \mathrm{~kb}$ (Human Genome Sequencing Project NT 034890 sequence)) is highly fragmented into 65 exons, transcribed in a $10 \mathrm{~kb}$
mRNA that encodes a 2871 amino acid protein. ${ }^{10,11,13,14}$ Three additional alternatively-spliced exons, likely untranslated, were found upstream of exon $1 .^{15}$ Conservation of nucleotide sequences within this region between human, mouse and porcine suggests that this region of the gene may harbour important regulatory elements. This region is GC-rich, contains a $\mathrm{CpG}$ island, and lacks conventional TATA or CCAAT boxes.

The deduced primary structure reveals a highly repetitive protein that contains essentially three repeated modules (Figure 1):

- The first repeated module is the EGF-like module that is homologous to one found in the Epidermal Growth Factor. These modules contain six cysteine residues that form three intra-domain disulfide bonds. There are 47 of these throughout the fibrillin-1 protein. Among these, 43 contain a conserved consensus sequence for calcium binding and are called cb EGF-like modules. In these domains, the residues putatively involved in calcium binding are numbered sequentially in Figure 2 as in Dietz and Pyeritz. ${ }^{16}$ They include the aspartic acid at position 2, glutamic acid at position 5, asparagine at position 10 and tyrosine or phenylalanine at position 15 .

- The second repeated module, found seven times interspaced with cb EGF-like in the protein, is called TGF $\beta 1$-binding protein-like module (TGF $\beta 1$-BP-like module) since it is homologous to modules found in the Transforming Growth Factor- $\beta 1$ binding protein. This domain appears to be limited to proteins that localise to matrix fibrils (fibrillins and latent transforming growth factor $\beta$-binding proteins (LTBPs)). These modules contain eight cysteine residues. The fourth TGF- $\beta 1$-BP-like module contains the RGD sequence which can interact with cell receptors. ${ }^{17}$ No specific function has yet been ascribed to these modules. However, some evidence suggests that these domains mediate specific protein-protein interactions. $^{18}$

- Finally, the protein contains a third module consisting of approximately 65 amino acids, and found twice in the protein. These are called 'hybrid modules' since they combine features of the EGF-like and the TGF- $\beta 1$-BP-like modules. This module is also found in LTBPs, which have a single hybrid domain.

Finally the protein contains three unique regions: a prolinerich region that may act as a 'hinge-like' region ${ }^{13}$ and the amino and carboxy terminal domains. The $\mathrm{N}$ - and C-terminal domains of the fibrillins display two prominent features: the presence of an even number of cysteine residues, four in the N-terminal and two in the C-terminal and the presence of the basic consensus sequence for processing by furin-types enzymes $\mathrm{BXBB}$ ( $\mathrm{B}=$ basic amino acid residue, $\mathrm{K}$ or $\mathrm{R}$ ) in each domain. The 4-cysteine domain in the N-terminus of fibrillins is homologous to 

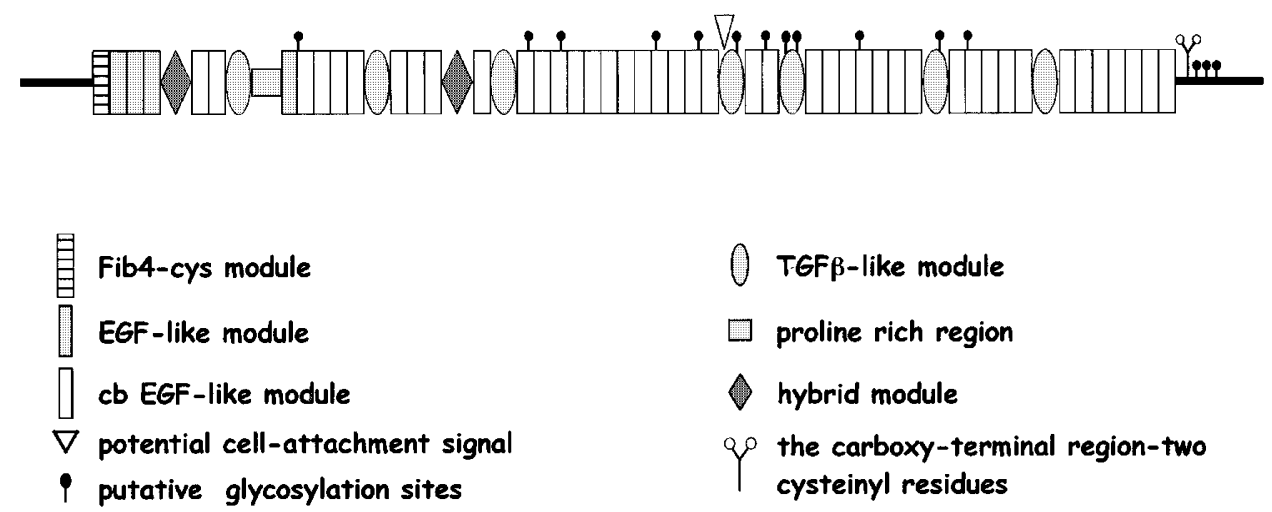

Figure 1 Schematic representation of the deduced primary structure of fibrillin-1.

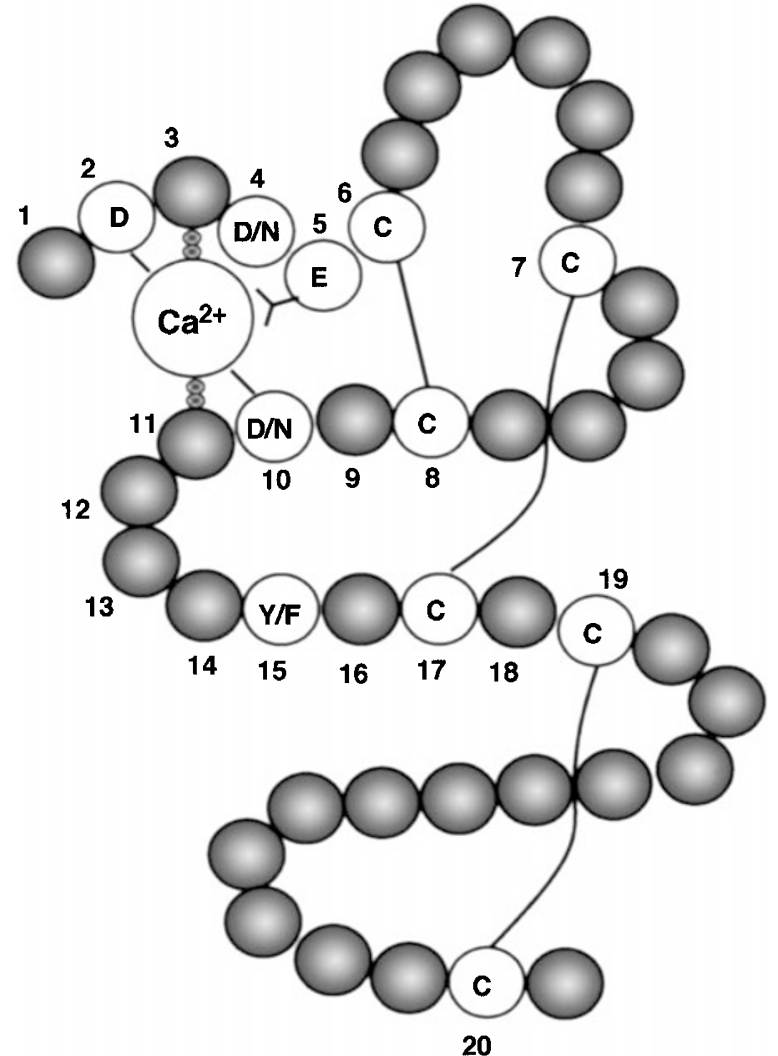

Figure 2 Schematic diagram of a normal cb EGF-like module. The cysteine residues which are disulfide-bonded and stabilize the native fold of the domain are represented in white. Other highly conserved residues are designated by their single-letter amino acid code. Residues with putative significance for calcium binding are numbered sequentially as in Dietz and Pyeritz. ${ }^{17}$

similar 4-cysteine domains in the N-terminal extended forms of the LTBPs. The C-terminal domains of the fibrillins are homologous to the C-terminal domain of all four members of the fibulin family, and thus a new type of extracellular module of approximately 120 amino acid residues in length has been proposed. ${ }^{19}$ This type of homology is not shared by the LTBPs.

When the FBN1 gene was cloned, a second gene sharing a high degree of homology was identified and located on chromosome 5. This gene was named FBN2 and the protein it encodes fibrillin-2. ${ }^{10}$ FBN2 has been genetically linked to a rare disorder that shares features of Marfan syndrome: congenital contractural arachnodactyly (CCA) (OMIM 120150). The clinical manifestations of CCA are essentially found in the skeleton and associated with distinctive manifestations including crumpled ears and campodactyly. Several mutations were identified in this gene in CCA patients. ${ }^{20}$

Ikegawa et al. described the structure and chromosomal assignment to 2 p16 of a 'fibrillin-like' gene (FBNL), that is highly homologous to fibrillin. ${ }^{21}$ The FBNL gene is expressed in many tissues but it is not expressed in brain and lymphocytes. The amino acid sequence of the FBNL gene is $36.3 \%$ identical to FBN1 (OMIM 134797) and $35.4 \%$ identical to FBN2. FBNL contains one EGF-like module and five repeated cb EGF-like modules. The gene spans approximately $18 \mathrm{~kb}$ of genomic DNA and contains 12 exons. In 1999, Stone et al. identified a single nonconservative mutation in the FBNL gene, also named EFEMP1 (EGF-containing fibulin-like extracellular matrix protein 1) in five families with Doyne honeycomb retinal dystrophy (DHRD; OMIM 126600), or malattia Leventinese (MLVT). ${ }^{22}$ This autosomal dominant disease is characterised by yellow-white deposits known as drusen that accumulate beneath the retinal pigment epithelium.

\section{The fibrillin proteins}

The fibrillins are extracellular matrix glycoproteins that show a wide distribution in both elastic and non-elastic tissues and are integral components of $10 \mathrm{~nm}$ diameter microfibrils. ${ }^{7,23}$ Fibrillin-1 is synthesised as profibrillin and proteolytically processed to fibrillin. The cleavage site has been mapped to the carboxy-terminal domain of profibril- 
lin-1 at position $\mathrm{S}^{2732}$ directly C-terminal to the $\mathrm{R}^{2728} \mathrm{KRR}$ sequence. Wild type profibrillin is not incorporated into extracellular matrix until it is converted to fibrillin. ${ }^{24}$ The $\mathrm{N}$-terminal region of each protein directs the formation of homodimers within a few hours after secretion and disulphide bonds stabilize the interaction. ${ }^{25}$ Dimer formation occurs intracellularly, suggesting that the process of fibrillin aggregation is initiated early after biosynthesis of the molecules. Fibrillin is post-translationally modified by $\beta$ hydroxylation and $\mathrm{N}$-and O-linked carbohydrate formation. $^{26}$

The solution structure of the TGF- $\beta$-like module from human fibrillin-1 identified a novel fold which was globular in nature ${ }^{27}$ and appears to break up linear regions within fibrillin-1 molecules after rotary shadowing electron microscopy. If these linker regions are effectively flexible, the kinks and bends observed in fibrillin-1 molecules would be required for proper alignment of molecules within the assembled microfibril. ${ }^{18}$

Baldock et al. have derived a model of fibrillin alignment in microfibrils based on automated electron tomography, immunolocalisation in directionally orientated untensioned microfibrils, mass changes on microfibril extension, immunofluorescence studies and published observations. ${ }^{28}$ Their model predicts maturation from a parallel head-to-tail alignment to an approximate one-third stagger that is stable as a 56-nm folded form, but not as an $\sim 100-\mathrm{nm}$ form. This model accounts for all microfibril structural features, suggests that inter- and intramolecular interactions drive conformation changes to form extensible microfibrils, and defines the number of molecules in cross section.

Fibrillin-1 and - 2 co-distribute in elastic and non-elastic connective tissues of the developing embryo, with a preferential accumulation of the FBN2 gene product in elastic fiber-rich matrices. ${ }^{23}$ Mouse study of the developmental expression of the fibrillin genes has revealed different patterns. Except for the cardiovascular system, in which Fbn1 gene activity is early and always higher than Fbn2, Fbn2 transcripts appear earlier than Fbn1 transcripts and accumulate for a short period of time just before overt tissue differentiation, i.e., a window of time immediately preceding elastogenesis. In contrast, the amount of Fbn1 transcripts increases at an apparently gradual rate throughout morphogenesis and is mainly expressed during late morphogenesis and well-defined organ structures. Furthermore, Fbn1 transcripts are predominantly represented in stress- and load-bearing structures like aortic adventitia, suspensory ligament of the lens, and skin. Spatio-temporal patterns of gene expression thus suggest distinct but related roles in microfibril physiology. Fibrillin-1 would provide mostly force-bearing structural support whereas fibrillin-2 would predominantly regulate the early process of elastic fibre assembly. ${ }^{29}$ Fibrillins would contribute to the structural and functional heterogeneity of microfibrils.

\section{Role of $\mathrm{Ca}^{2+}$ in fibrillin}

The implication of the variable calcium binding affinities observed in fibrillin fragments is biologically significant. A number of studies have shown that the presence of calcium ions significantly protects full-length or recombinant fragments of fibrillin-1 from proteolysis by trypsin, elastase, endoproteinase Glu-C, plasmin and matrix metalloproteinases. ${ }^{30-34}$ Moderate to high affinities for calcium suggest that fibrillin cb EGF-like modules would be close to fully saturated in vivo. Particular regions of fibrillin may need to be rigid for appropriate function. For example, cb EGFlike 12-13, located in the neonatal Marfan syndrome region (see Pathogenic mechanisms), where mutations leading to severe phenotypes cluster, may be part of a region where rigidity is required for function. Fully saturated calcium binding sites may be required for stabilisation of the microfibril against proteolytic degradation, when lowaffinity sites not fully saturated in vivo may contribute to flexibility of the polypeptide chain or to biomechanical function. It may be advantageous to allow some degree of extensibility of assembled microfibrils in tissues subjected to mechanical forces. The importance of domain context for modulating the structural effects of calcium binding mutations suggests an explanation why MFS phenotypes associated with apparently similar mutations may be diverse. ${ }^{33}$

\section{FBN1 gene mutations in Marfan syndrome and related disorders}

To date over 500 mutations have been identified in the FBN1 gene in Marfan syndrome patients and related diseases (Figure 3) ${ }^{34}$ (Collod-Béroud et al., In preparation). No major rearrangements have been identified except for three cases of multi-exon deletions. ${ }^{35,36}$ Three categories of mutations have been described: (1) missense mutations, (2) small insertions or deletions, mutations causing premature termination of translation and (3) exon-skipping mutations.

FBN1 gene mutations have been identified in complete and incomplete forms of Marfan syndrome but also in various disorders: severe neonatal Marfan syndrome, dominantly inherited ectopia lentis, ${ }^{37}$ isolated skeletal features of MFS, $^{38}$ the Shprintzen-Goldberg syndrome ${ }^{39}$ and, more recently, familial or isolated forms of aortic aneurysms. ${ }^{40}$ These results define the new molecular group of 'type 1 fibrillinopathies' that comprises a spectrum of overlapping diseases. Presently no definite genotype/phenotype correlations have been identified except for neonatal mutations (see pathogenic mechanisms). To facilitate their identification, a 'Marfan database' has been developed that includes not only molecular but also clinical data. The database is attached to a software that provides various tools for its analysis and allows optimised multicriteria 


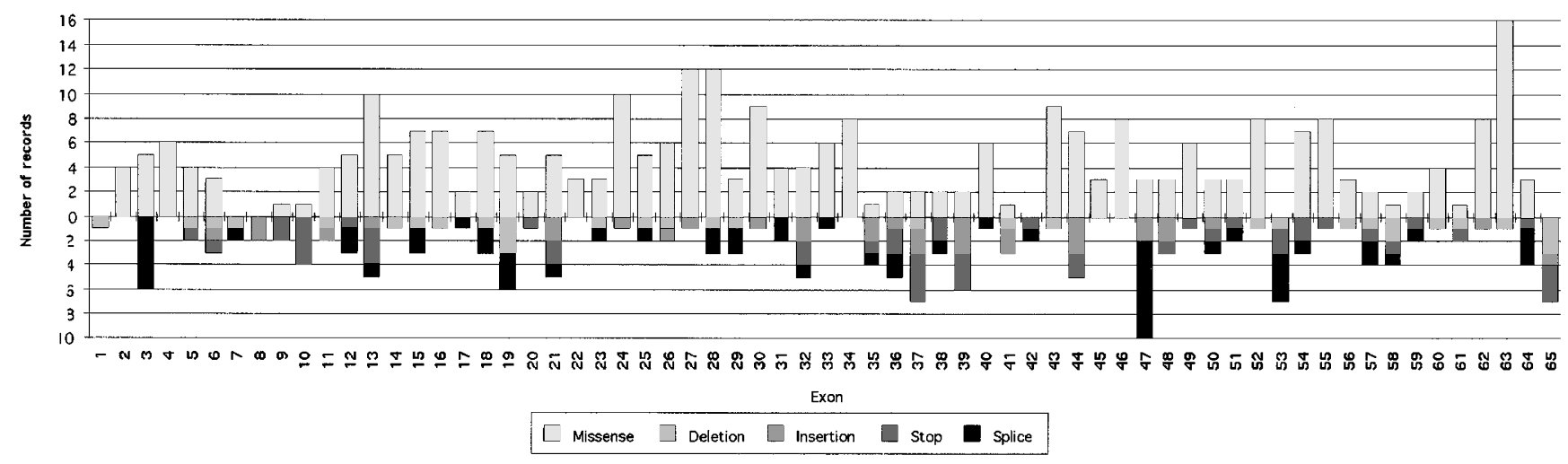

Figure 3 Distribution of the mutations identified in FBN1 gene.

research. ${ }^{34,41-43}$ It is only through a large collaborative international effort that genotype/phenotype correlations will eventually be identified.

Nonpenetrance has never been demonstrated for families in which patients carrying fibrillin-1 mutations are associated with Marfan syndrome. However, patients with the same mutation can show a wide degree of phenotypic variability. This has been exemplified in large pedigrees with sharp differences in clinical severity of musculoskeletal and cardiovascular features of the syndrome. ${ }^{44}$

\section{Neonatal Marfan syndrome and FBN1 gene mutations}

Neonatal Marfan syndrome is the most severe form of the disorder. Affected new-borns display severe cardiac valve regurgitation and dilatation of the proximal aorta which usually lead to heart failure and death in the first year of life. Skeletal manifestations such as arachodactyly, dolichostenomelia, and pectus deformities are typically present. Such infants may also display congenital flexion contractures, crumpled ears, loose redundant skin, and a characteristic 'senile' facial appearance. ${ }^{45}$ The mean life span is usually low (approximately 1 year $^{46}$ ). The primary cause of death is congestive heart failure associated with mitral and tricuspid regurgitation. Family investigation usually reveals that the Marfan patients with the severe neonatal phenotype are sporadic cases: Buntinx et al. reported that 37 of 44 cases with neonatal manifestations were sporadic. ${ }^{45}$ For a long time it was generally thought that the neonatal phenotype could be explained by mutations in a distinct gene than that involved in the classic 'adolescent-adult' form of the syndrome as the observed symptoms were extremely severe and overlapped with congenital contractural arachnodactyly. Godfrey et al. showed an abnormal morphology of fibrillin microfibrils in fibroblast cultures from patients with the neonatal phenotype. ${ }^{46}$ As in the classic 'adolescent-adult' form, there was an apparent decrease in accumulation of immunostainable fibrillin, but they appeared shorter, fragmented and frayed. Molecular analyses revealed that the neonatal Marfan syndrome was also due to mutations within the FBN1 gene. Furthermore a clustering of mutations in the protein region encoded by exons 24-32 was observed (Figure 4), suggesting an unknown but critical function of these domains. ${ }^{47}$ The severe phenotype associated with these specific mutations in this region of the gene represents, to date, the only loose genotype/phenotype relationship established. The observed clustering of mutations enables, in a first step, direct screening of this region of the FBN1 gene to help in diagnosis of neonatal Marfan syndrome in patients. Finally, confirmation of the sporadic nature of the mutation is important for genetic counseling since perinatal lethal Marfan syndrome can also result from compound heterozygosity ${ }^{48}$ or potential homozygosity.

\section{Pathogenic mechanisms}

Fibrillins are important components of the microfibrillar system that may act as a scaffold for elastogenesis. Elastic fibres first appear in foetal development as aggregates of microfibrils. These microfibrils are arranged in parallel arrays on which elastin is deposited and appears as an amorphous material. Elastin-containing microfibrillar bundles aggregate to form true elastic fibres. These observations suggest that microfibrils determine the form and the orientation of elastic fibres, therefore directing fibre assembly as a scaffold on which elastin is deposited. ${ }^{29}$ This model explains the typical fragmentation and disarray of elastic fibres observed in the media of Marfan patients. However, unlike elastin, fibrillin-1 is also highly expressed in the vascular adventia. Therefore reduction of this protein in the adventia is very likely also involved in the mechanism for dilatation and for increased risk of aneurysm since the role of the adventia is to maintain the vascular diameter. The pleiotropic manifestations of the disease can be explained by the observation that numerous microfibrillar 


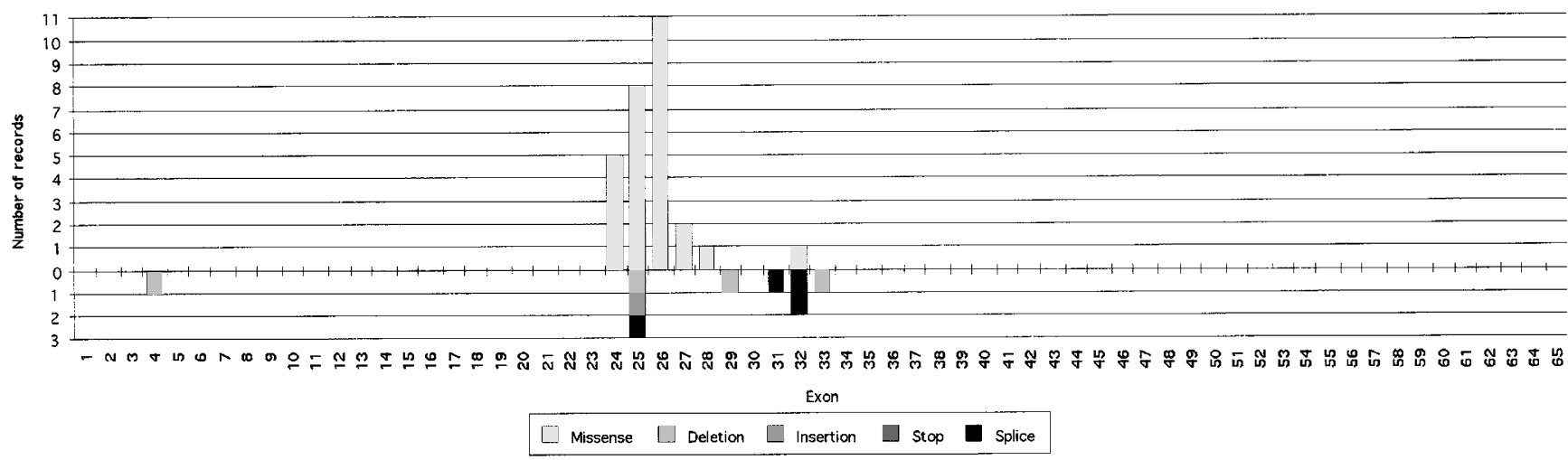

Figure 4 Distribution of mutations identified in FBN1 gene associated with a neonatal form of Marfan syndrome.

aggregates devoid of elastin are found in the zonule, as well as cartilage and the extracellular matrix of many organs. However, the actual pathogenic mechanisms in these tissues still remain speculative.

At the molecular level, two different groups of mutations are distinguishable: mutations leading to a shortened protein and missense mutations. The first group correspond to one third of the mutations and is constituted of nonsense mutations ( $\sim 10 \%$ of all mutations), splicing errors $(\sim 12 \%$, only one demonstrated case of exon addition), small deletions leading to premature STOP codon ( $\sim 8 \%)$, small inframe deletions $(\sim 2 \%)$, multi-exon deletions $(\sim 0.6 \%)$, and insertions leading to premature STOP codon $(\sim 4 \%)$. Mutations can be responsible for the appearance of a premature STOP codon that reduces the stability of the mutant transcript and consequently greatly reduces protein production from the mutated copy of the gene (in the affected subjects, the amount of fibrillin-1 protein produced is $50 \%$ that of normal and is produced only from the normal gene copy), or for the production from the mutated copy of an abnormal monomere that considerably interferes with the assembly (polymerization) of fibrillin molecules (the amount of fibrillin is greatly reduced, $<35 \%)$. The second group represent two-thirds of mutations and correspond to missense mutation. Among them, three-quarters are located in calcium binding modules. They are implicated either in creating ( $\sim 3 \%$ of all mutations) or substituting $(\sim 24 \%)$ cysteine residues potentially implicated in disulfide bonding and consequently in the correct folding of the monomere. The majority of remaining mutations of this type of module affects residues of the calcium consensus sequence that plays a major role in defining interdomain linkage. ${ }^{49}$ An increased protease susceptibility is a mechanism also suggested for missense mutations. Other modules are carriers of one quarter of missense mutations and pathological mechanisms have yet to be clearly demonstrated.

What is still unknown are the multiple consequences triggered by the various mutations and the effect of unknown modifier (enhancing or protecting) genes on the clinical expression. These mechanisms and the great number of mutations identified in the FBN1 gene explain the great variability of the disease observed not only between families but also among affected individuals in a single family.

\section{Genetic heterogeneity in Marfan syndrome}

The clinical variability of Marfan syndrome is only partly explained by the great number of mutations identified in the FBN1 gene. In effect, we have demonstrated the existence of genetic heterogeneity, ie the involvement, in certain cases of Marfan syndrome of mutations located in another gene named MFS2 (for Marfan syndrome type 2). Genetic heterogeneity was demonstrated through the study of a large French family in which affected individuals display an incomplete form of the syndrome: typical skeletal and cardiovascular features as well as involvement of the skin and integument. No ocular manifestations were observed until recently when one of the children developed ectopia lentis. We showed that fibrillin-1 was normal in several affected family members and excluded linkage between the FBN1 gene and the disease in the family. ${ }^{50}$ By exclusion mapping we located the MFS2 gene on the short arm of chromosome $3 .^{51}$ In this area is located the gene that encodes fibuline-2 (FBLN2), another microfibrillar component. Again through a double approach (genetic and protein) we showed that MFS2 and FBLN2 were not identical. $^{52}$ We are now identifying MFS2 through positional cloning. Other teams have already identified families comparable to the French family in that they are not linked to or do not carry a mutation in the FBN1 gene (M Boxer, L Peltonen and B Steinmann, personal communications). Clinically these families are indistinguishable from other families linked to FBN1. Therefore, we are also trying to determine the percentage of Marfan syndrome cases that are associated with mutations in MFS2 through genetic analyses as well as their clinical spectrum. Other teams, through protein studies have identified between seven 
and $16 \%$ of Marfan syndrome patients with normal fibrillin metabolism. ${ }^{53,54}$ The precise determination of this per cent is important for laboratories involved in diagnosis of Marfan syndrome since it will give the risk associated with investigation of only the FBN1 gene.

\section{Animal model}

The first animal model described was a limousine calf which presented with skeletal (kyphosis, long, thin limbs), integuments (severe joint and tendon laxity), ocular (microspherophakia, ectopia lentis) and cardiovascular (heart murmurs, aortic dilatation, sudden death at a young age due to aortic rupture) abnormalities. ${ }^{55}$ The similarities between the human and the bovine diseases suggest that similar metabolic defects could be responsible. To date, although reduced immunostained fibrillin in cultured aortic smooth muscle cells in this limousine calf, ${ }^{56}$ no mutation in the corresponding bovine FBN1 gene or in another gene was yet identified in this model.

Mice carrying the Tight skin (Tsk) mutation harbor a genomic duplication within the fibrillin-1 (Fbn1) gene that results in a larger than normal in-frame $F b n 1$ transcript. ${ }^{57}$ $T s k /+$ mice exibit a thickening of the skin with loss of elasticity, larger skeletal size because of excessive bone and cartilage growth, emphysema-like condition, myocardial hypertrophy and small tendons with tendon sheath hyperplasia. Tsk fibrillin-1 is produced, assembled, and deposited in the extracellular matrix but beaded Tsk fibrillin-1 microfibrils have a longer than normal periodicity and an altered morphology and organisation in skin. Vascular complications were thought to be absent in these animals because the level of functional microfibrils does not drop below the critical threshold. The heterozygous mice have a normal life span contrary to the human counterpart.

Gene-targeting experiments in mice resulted in two mutant lines in mice: the $\mathrm{mg} \Delta$ mutant from the $\mathrm{J} 1$ lines of ES cells (deletion of exons $19-24)^{58}$ and the mgR mutant from R1 lines of ES cells (integration of the PGKneo-cassette without loss of endogenous sequence). ${ }^{59}$ Homozygous mg $\Delta$ mice begin life with a drastic reduction in protein $(5 \%)$ and die early because of structural failure of the vascular system. Homozygous mgR mice produce a quarter of the normal amount of fibrillin-1 and display phenotypic features in the skeleton and the aorta similar to those of patients with classic Marfan syndrome. The $\mathrm{mgR} / \mathrm{mgR}$ mice support the notion that microfibrils control bone overgrowth negatively.

Finally, Jaubert et al. demonstrated the implication of type $\mathrm{C}$ receptor for natriuretic peptides (NPR-C) in the strigosus (stri) mutation. ${ }^{60}$ Homozygous mutant mice show as early as 6 days of age increased body length, longer digits, and a typical cone-shaped implantation of the tail. When older, mutant mice are exceptionally thin and have arachnodactyly, thoracic kyphosis and frequent tail and/or sacral kinks. The unexpected expression of mutations with- in this gene as a Marfan-like skeletal phenotype should not be overlooked in the investigation of the pathogenesis of Marfan syndrome.

\section{Marfan syndrome is still an essentially clinical diagnosis}

Although no specific therapy exists for Marfan syndrome, it is of great importance to confirm or firmly exclude the diagnosis in family members at risk as early as possible because of the potential fatal complications of the disease. At present, diagnosis is still based on thorough clinical examination, including measurements of body proportions, echocardiography of the aorta, slit-lamp ophthalmological evaluation and radiographs. A complete family history is also an essential part of the diagnosis. However in some cases the manifestations are not evident until adolescence and the clinical expression of the disease varies greatly between affected members of a single family. Therefore, there is an absolute need for an accurate diagnostic test.

The discovery of the involvement of fibrillin- 1 has raised high hopes for a protein or DNA test applicable to Marfan syndrome patients. Immunofluorescence studies of cultured fibroblasts and skin sections of patients using monoclonal antibodies against fibrillin have revealed that the amount of fibrillin deposition or of fibrillin microfibrils is greatly reduced. ${ }^{8}$ Therefore, immunofluorescence analysis could be helpful in diagnosis. However the method has proven to be insufficiently sensitive and specific because of the existence of non-Marfan syndrome type 1 fibrillinopathies and of genetic heterogeneity. Therefore, an abnormal test result does not diagnose Marfan syndrome, and a normal test result does not exclude Marfan syndrome.

The identification of the FBN1 gene has allowed the development of two types of diagnostic tests: either genetic family studies or mutation identification. Family studies can be performed with specific FBN1 polymorphic markers to identify the mutation-bearing haplotype. ${ }^{61}$ These studies are only reliable in families in which several affected individuals are available since the involvement of a FBN1 mutation (and not that of another gene) must be clearly demonstrated. However, most family structures do not comply with this requirement. Furthermore, the method is inappropriate in sporadic cases. In practice, these instances represent over $40 \%$ of the cases referred for biological diagnosis. The second molecular test is mutation identification. Mutation identification is very costly and long. In effect, there is no quick and $100 \%$ reliable method to investigate a large $(\sim 230 \mathrm{~kb})$ and highly fragmented (10 kb of coding sequence fragmented in 65 exons) gene, knowing that almost each family has its own specific defect and that the mutations are essentially point mutations. Finally, this very costly analysis may fail to identify a mutation since only the coding sequence and closely surrounding regions are investigated. However, in the case of neonatal Marfan syndrome, where a clustering of muta- 
tions is found in a specific region, molecular diagnosis can be performed. In all other instances and until better molecular tools are available, mutation identification cannot be performed on a systematic basis. However, in a few cases where the family mutation had been identified, it was possible to perform prenatal diagnosis on chorionic villus samples or offer presymptomatic diagnosis in children at risk of affected subjects. ${ }^{62,63}$

\section{References}

1 Marfan A: Un cas de déformation congénitale des quatre membres, plus prononcée aux extrémités, caractérisée par l'allongement des os avec un certain degré d'amincissement. Bull Mém Soc Méd Hôp Paris 1896; 13: 220-227.

2 Beighton P, de Paepe A, Danks D et al: International nosology of heritable disorders of connective tissue. Am J Med Genet 1988; 29: $581-594$.

3 De-Paepe A, Devereux R, Dietz H, Hennekam R, Pyeritz R: Revised diagnostic criteria for the Marfan syndrome. Am J Med Genet 1996; 62: $417-426$.

4 Rose P, Levy H, Ahn N et al: A comparison of the Berlin and Ghent nosologies and the influence of dural ectasia in the diagnosis of Marfan syndrome. Genetics in medicine 2000; 2: 278-282.

5 Weve H: Über Arachnodaktylie (dystrophia mesodermalis congenita, Typus Marfan). Archiv Augenheilk 1931; 104: 1-46.

6 McKusick VA: The cardiovascular aspects of Marfan's syndrome: A heritable disorder of connective tissue. Circulation 1955; 11: 321 341.

7 Sakai L, Keene D, Engvall E: Fibrillin, a new 350 kD glycoprotein is a compound of extracellular microfibrils. J Cell Biol 1986; 103: 2499-2509.

8 Hollister D, Godfrey M, Sakai L, Pyeritz R: Immunohistologic abnormalities of the microfibrillar system in the Marfan syndrome. N Eng J Med 1990; 323: $152-159$.

9 Dietz H, Cutting G, Pyeritz R et al: Marfan syndrome caused by a recurrent de novo missense mutation in the fibrillin gene. Nature 1991; 352: 337-339.

10 Lee B, Godfrey M, Vitale E et al: Linkage of Marfan syndrome and a phenotypically related disorder to two different fibrillin genes. Nature 1991; 352: 330-334.

11 Maslen C, Corson G, Maddox B, Glanville R, Sakai L: Partial sequence of a candidate gene for the Marfan syndrome. Nature 1991; 352: 334-337.

12 Kainulainen K, Pulkkinen L, Savolainen A, Kaitila I, Peltonen L: Location on chromosome 15 of the gene defect causing Marfan syndrome. N Eng J Med 1990; 323: 935-939.

13 Corson G, Chalberg S, Dietz H, Charbonneau N, Sakai L: Fibrillin binds calcium and is coded by cDNAs that reveal a multidomain structure and alternatively spliced exons at the 5 ' end. Genomics 1993; 17: 476-484.

14 Pereira L, d'Alessio M, Ramirez F et al: Genomic organization of the sequence coding for fibrillin, the defective gene product in Marfan syndrome. Hum Mol Genet 1993; 2: 961-968.

15 Biery N, Eldadah Z, Moore C, Setten G, Spencer F, Dietz H: Revised genomic organization of $F B N 1$ and significance for regulated gene expression. Genomics 1999; 56: 70-77.

16 Dietz HC, Pyeritz RE: Mutations in the human gene for fibrillin-1 (FBN1) in the Marfan syndrome and related disorders. Hum Mol Genet 1995; 4: 1799-809, Review.

17 Sakamoto H, Brockelmann T, Cheresh D, Ramirez F, Rosenbloom J, Mecham R: Cell-type specific recognition of RGD- and nonRGD-containing cell binding domains in fibrillin-1. J Biol Chem 1996; 271: 4916-4922.
18 Handford PA, Downing AK, Reinhardt DP, Sakai LY: Fibrillin: From domain structure to supramolecular assembly. Matrix Biol 2000; 19: 457-470.

19 Giltay R, Timpl R, Kostka G: Sequence, recombinant expression and tissue localization of two novel extracellular matrix proteins, fibulin-3 and fibulin-4. Matrix Biol 1999; 18: 469-480.

20 Wang M, Clericuzio CL, Godfrey M: Familial occurrence of typical and severe lethal congenital contractural arachnodactyly caused by missplicing of exon 34 of fibrillin-2. Am J Hum Genet 1996; 59: $1027-1034$

21 Ikegawa S, Toda T, Okui K, Nakamura Y: Structure and chromosomal assignment of the human S1-5 gene (FBNL) that is highly homologous to fibrillin. Genomics 1996; 35: 590-592.

22 Stone EM, Lotery AJ, Munier FL et al: A single EFEMP1 mutation associated with both malattia Leventinese and Doyne honeycomb retinal dystrophy. Nature Genetics 1999; 22: 199-202.

23 Zhang H, Apfelroth S, Hu W et al: Structure and expression of fibrillin-2 a novel microfibrillar component preferentially located in elastic matrices. J Cell Biol 1994; 124: 855-863.

24 Raghunath M, Putnam EA, Ritty T et al: Carboxy-terminal conversion of profibrillin to fibrillin at a basic site by PACE/furin-like activity required for incorporation in the matrix. J Cell Science 1999; 112: $1093-1100$.

25 Trask TM, Ritty TM, Broekelmann T, Tisdale C, Mecham RP: Nterminal domains of fibrillin 1 and fibrillin 2 direct the formation of homodimers: a possible first step in microfibril assembly. Biochem J 1999; 340: 693-701.

26 Glanville RW, Qian RQ, McClure DW, Maslen CL: Calcium binding, hydroxylation, and glycosylation of the precursor epidermal growth factor-like domains of fibrillin-1, the Marfan gene protein. J Biol Chem 1994; 269: 26630-26634.

27 Yuan X, Downing AK, Knott V, Handford PA: Solution structure of the transforming growth factor $\beta$-binding protein-like module, a domain associated with matrix fibrils. EMBO J 1997; 16: 66596666.

28 Baldock C, Koster A, Ziese U et al: The supramolecular organization of fibrillin-rich microfibrils. J Cell Biol 2001; 152: 1045 1056.

29 Zhang H, Hu W, Ramirez F: Developmental expression of fibrillin genes suggests heterogeneity of extracellular microfibrils. $J$ Cell Biol 1995; 129: 1165-1176.

30 Reinhardt DP, Ono RN, Sakai LY: Calcium stabilizes fibrillin-1 against proteolytic degradation. J Biol Chem 1997; 272: 1231 1236.

31 Reinhardt DP, Ono RN, Notbohm H, Muller PK, Bachinger HP, Sakai LY: Mutations in calcium-binding epidermal growth factor modules render fibrillin-1 susceptible to proteolysis. A potential disease-causing mechanism in Marfan syndrome. I Biol Chem 2000; 275: $12339-12345$.

32 Ashworth J, Murphy G, Rock M et al: Fibrillin degradation by matrix metalloproteinases: Implications for connective tissue remodeling. Biochem J 1999; 340: 171-181.

33 McGettrick AJ, Knott V, Willis A, Handford PA: Molecular effects of calcium binding mutations in Marfan syndrome depend on domain context. Hum Mol Genet 2000; 9: 1987-1994.

34 Collod-Béroud G, Béroud C, Adès L et al: Marfan Database (third edition): new mutations and new routines for the software. Nucleic Acids Res 1998; 26: 229-233.

35 Kainulainen K, Sakai LY, Child A et al: Two mutations in Marfan syndrome resulting in truncated fibrillin polypeptides. Proc Nat Acad Sci USA 1992; 89: 5917-5921.

36 Liu W, Schrijver I, Brenn T, Furthmayr H, Francke U: Multi-exon deletions of the FBN1 gene in Marfan syndrome. BMC Med Genet 2001; 2: 11-19.

37 Kainulainen K, Karttunen L, Puhakka L, Sakai L, Peltonen L: Mutations in the fibrillin gene responsible for dominant ectopia lentis and neonatal Marfan syndrome. Nature Genetics 1994; 6 : 64-69. 
38 Milewicz D, Grossfield J, Cao SN, Kielty C, Covitz W, Jewett T: A mutation in $F B N 1$ disrupts profibrillin processing and results in isolated skeletal features of the Marfan syndrome. J Clin Invest 1995; 95: $2373-2378$.

39 Sood S, Eldadah Z, Krause W, McIntosh I, Dietz H: Mutation in fibrillin-1 and the Marfanoid-craniosynostosis (Shprintzen-Goldberg) syndrome. Nature Genetics 1996; 12: 209-211.

40 Milewicz D, Michael K, Fisher N, Coselli J, Markello T, Biddinger A: Fibrillin-1 (FBN1) mutations in patients with thoracic aortic aneurysms. Circulation 1996; 94: 2708-2711.

41 Collod G, Béroud C, Soussi T, Junien C, Boileau C: Software and database for the analysis of mutations in the human FBN1 gene. Nucl Acids Res 1996; 24: 137-140.

42 Collod-Béroud G, Béroud C, Adès L et al: Marfan database (second edition): Software and database for the analysis of mutations in the human FBN1 gene. Nucl Acids Res 1997; 25: 147-150.

43 Beroud C, Collod-Beroud G, Boileau C, Soussi T, Junien C: UMD (Universal mutation database): A generic software to build and analyze locus-specific databases. Hum Mutat 2000; 15: 86-94.

44 Dietz HC, Pyeritz RE, Puffenberger EG et al: Marfan phenotype variability in a family segregating a missense mutation in the epidermal growth factor-like motif of the fibrillin gene. J Clin Invest 1992; 89: 1674-1680.

45 Buntinx I, Willems P, Spitaels S, VanReempst P, DePaepe A Dumon J: Neonatal Marfan syndrome with congenital arachnodactyly, flexion contractures, and severe cardiac valve insufficiency. J Med Genet 1991; 28: 267-273.

46 Godfrey M, Raghunath M, Cisler J et al: Abnormal morphology of fibrillin microfibrils in fibroblast cultures from patients with neonatal Marfan syndrome. Am J Pathol 1995; 146: 1414-1421.

47 Putnam E, Cho M, Zinn A, Towbin J, Byers P, Milewicz D: Delineation of the Marfan phenotype associated with mutations in exons 23-32 of the FBN1 gene. Am J Med Genet 1996; 62: 233-242.

48 Karttunen L, Raghunath M, Lonnqvist L, Peltonen L: A compound heterozygous Marfan patient: Two defective fibrillin alleles result in a lethal phenotype. Am J Hum Genet 1994; 55 $1083-1091$.

49 Knott V, Downing A, Cardy C, Handford P: Calcium binding properties of an epidermal growth factor-like domain pair from human fibrillin-1. J Mol Biol 1996; 255: 22 - 27.

50 Boileau C, Jondeau G, Babron MC et al: Familial Marfan-like aortic dilatation and skeletal anomalies are not linked to the fibrillin genes. Am J Hum Genet 1993; 53: 46-57.
51 Collod G, Babron MC, Jondeau G et al: A second locus for Marfan syndrome maps to chromosome 3p24.2-p25. Nature Genetics 1994; 8: 264-268.

52 Collod G, Chu ML, Sasaki T et al: Fibuline-2: Genetic mapping and exclusion as a candidate in Marfan syndrome type 2. Eur $J$ Hum Genet 1996; 4: 292-295.

53 McGookey-Milewicz D, Pyeritz R, Crawford ES, Byers P: Marfan syndrome: Defective synthesis, secretion, and extracellular matrix formation of fibrillin by cultured dermal fibroblasts. J Clin Invest 1992; 89: 79-86.

54 Aoyama T, Francke U, Dietz H, Furthmayer H: Quantitative differences in biosynthesis and extracellular deposition of fibrillin in cultured fibroblasts distinguish five groups of Marfan syndrome patients and suggest distinct pathogenic mechanisms. J Clin Invest 1994; 94: 130-137.

55 Besser TE, Potter KA, Bryan GM, Knowlen GG: An animal model of the Marfan syndrome. Am J Med Genet 1990; 37: 159-165.

56 Potter KA, Hoffman Y, Sakai LY, Byers PH, Besser TE, Milewicz DM: Abnormal fibrillin metabolism in bovine Marfan syndrome. Am J Pathol 1993; 142: $803-810$.

57 Kielty CM, Raghunath M, Siracusa L et al: The tight skin mouse: demonstration of mutant fibrillin-1 production and assembly into abnormal microfibrils. J Cell Biol 1998; 140: 1159-1166.

58 Pereira L, Andrikopoulos K, Tian J et al: Targetting of the gene encoding fibrillin-1 recapitulates the vascular aspect of Marfan syndrome. Nature Genetics 1997; 17: 218-222.

59 Pereira L, Lee SY, Gayraud B et al: Pathogenetic sequence for aneurysm revealed in mice underexpressing fibrillin-1. Proc Natl Acad Sci USA 1999; 96: 3819-3823.

60 Jaubert J, Jaubert F, Martin N et al: Three new allelic mouse mutations that cause skeletal overgrowth involve the natriuretic peptide receptor C gene (Npr3). Proc Natl Acad Sci USA 1999; 96: $10278-10283$.

61 Pereira L, Levran O, Ramirez F et al: A molecular approach to the stratification of cardiovascular risk in families with Marfan's syndrome. N Eng J Med 1994; 331: 148 - 153.

62 Godfrey M, Vandemark N, Wang M et al: Prenatal diagnosis and a donor splice site mutation in fibrillin in a family with Marfan syndrome. Am J Hum Genet 1993; 53: 472-480.

63 Rantamäki T, Raghunath M, Karttunen L, Lönnqvist L, Child A, Peltonen L: Prenatal diagnosis of Marfan syndrome: Identification of a fibrillin-1 mutation in chorionic villus sample. Prenat Diag 1995; 15: 1176-1181. 